\title{
The Evaluation of Innovation Talent Training in Universities Base on Ahp-Fuzzy
}

\author{
LIU LING ${ }^{1}$, HAN NA ${ }^{2}$ FU ZHAO-XIA ${ }^{3}$ \\ ${ }^{1}$ School of Humanities and Law, Hebei University of Science and Technology, Shijiazhuang 050018, China \\ ${ }^{2}$ School of Economy and Management, Hebei University of Science and Technology, Shijiazhuang 050018, China \\ ${ }^{3}$ Department of Science and Research, Hebei University of Science and Technology, Shijiazhuang 050018, China \\ E-MAIL:liulingpeiyu@126.com, hanna1974@126.com, fzx68@hebust.edu.cn
}

\begin{abstract}
In recent years, the question of innovation talent has become a major proposition of sustainable development in the world. As the main base of training innovation talents universities have still insufficient in evaluating innovation talents. This article refers to existing research results to build the evaluation indicator system of innovation talent training in universities from the point of input, conversion, output and applies AHP-fuzzy comprehensive evaluation model to evaluate the indicator system. It closely links to the theory and practice and provides a scientific method to measure the level of innovation talent training for our universities.
\end{abstract}

Keywords-University; innovation talent; AHP-fuzzy; evaluation

\section{INTRODUCTION}

After entering the new century, the question of innovation talents has became a major proposition of sustainable development in the world. Our country places innovation talent training in the key level if the great rejuvenation of Chinese nation can smoothly realize. Universities have many characteristics, such as talent accumulation, good infrastructure, free academic atmosphere, and the impact of multi-disciplinary. The characteristics make universities the fertile soil to generate new knowledge and new ideas, and are main base to train innovation talents of science and technology and product and disseminate technological knowledge. So how to ensure the training quality of innovation talents in universities is an important issues faced by universities. However, the evaluation of innovation talents training in universities is a comprehensive, multi-factors complex issue, and most factors are qualitative description. It makes digitalization on non-digitalized problems to establish an objective evaluation indicator system of innovation talents training. It can play a very good guide for the establishment of innovation talents training in universities. On the basis of domestic and foreign scholars research on innovation talents training in recent years the paper uses the method of AHP and fuzzy combination to hope to establish a more complete and effective indicator system. By the indicator system we can measure the level of innovation talents training in universities so as to continuously promote the universalization process of innovation talents training in our universities.

\section{THE DESIGN OF EVALUATION INDICATOR SYSTEM OF INNOVATION TALENTS TRAINING IN UNIVERSITIES}

\section{A. The construction principle of evaluation indicator system}

In order to fully and truly reflect the inner essence and composition of innovation talents training in universities and facilitate the comprehensive evaluation of innovation talents training in universities the paper should abide by the following principles when establishing the comprehensive evaluation index system of innovation talents training in universities.

(1) Scientific principle. It includes the accuracy and comprehensiveness of indicator system. The indicators can correctly reflect the connotation represented by the indicators themselves and fully reflect the process characteristics of innovation talents training in universities.

(2) Systematic principle. Each indicator should have a clear connotation and extension and form a certain logical relation between the indicators to make indicator system form an organic whole, which reflects the process systemic of innovation talents training in universities.

(3) The principle of comparability. Not only take into account the comparability between universities but also turn inappropriate indicators into comparable indicators when establishing the indicator system.

(4) Innovation principle. Around innovation talents training in universities and based on the process of innovation talents training and the comprehensive of training quality the paper establishes the comprehensive evaluation indicator system of innovation talents training in universities from the point of inputs, outputs and revenue of innovation talents training.

\section{B. The comprehensive evaluation indicator system of innovation talents training in universities}

Innovation talents training can not produce out of thin air and need the investment of universities, including teaching, research and other inputs and so on. These investments reflect existing basic conditions in universities, such as human, material, financial and policy regime and so on. By the joint efforts of teachers and managers the investments can create a number of results in the process of innovation talents training. To innovation talents training in universities the results express a facilitating role and ultimately take a positive 
impact on talents training to form the effectiveness of innovation talents training. Including a number of graduates with high-quality innovative consciousness, innovative spirit and innovative ability. Therefore, innovation talent training in universities is a process of input, conversion and output. If taking the strength of basis support as input, the force of the strength as conversion, the effect of innovation talents training as output, then this idea can be summarized as: relying on their own strength and inspired by a series of management system universities result in the vitality of innovation talents training, create a series of means and methods and apply them to education to train the innovation spirit and innovation ability of students. It is shown as the effect of innovation talents training by means of a certain way.

Based on the above analysis evaluation indicator system of innovation talents training in universities is shown in Table 1.

TABLE 1. EVALUATION INDICATOR SYSTEM OF INNOVATION TALENTS TRAINING IN UNIVERSITIES

\begin{tabular}{|c|c|c|}
\hline The First Level Indicators & The Second Level Indicators & The Third Level Indicators \\
\hline \multirow{12}{*}{ Basis support strength } & \multirow{3}{*}{ material basis } & the number of provincial key laboratory or above \\
\hline & & the number of provincial experimental demonstration center or above \\
\hline & & the number of laboratory equipment owned by average student \\
\hline & \multirow{4}{*}{ subject foundation } & the number of national key subject \\
\hline & & the number of provincial key subject \\
\hline & & the number of master degrees \\
\hline & & the number of doctor degrees \\
\hline & \multirow{5}{*}{ human resources } & faculty-student ratio \\
\hline & & the proportion of teachers with high professional titles \\
\hline & & the proportion of provincial teaching team \\
\hline & & the proportion of provincial scientific research team \\
\hline & & the proportion of provincial master teachers \\
\hline \multirow{12}{*}{ Basis support contribution } & \multirow{2}{*}{ material basis contribution } & the proportion of experimental design \\
\hline & & the proportion of laboratory opening \\
\hline & \multirow{5}{*}{ teaching contribution } & the proportion of bilingual course \\
\hline & & the proportion of multimedia teaching \\
\hline & & the number of provincial research teaching item or above by average teacher \\
\hline & & the number of provincial top-quality course \\
\hline & & the number of provincial teaching achievement prize \\
\hline & \multirow{5}{*}{ scientific research contribution } & the number of scientific research funds \\
\hline & & scientific and technical achievements \\
\hline & & the number of academic paper by indexed \\
\hline & & the number of books published by average teacher \\
\hline & & patent number \\
\hline \multirow{6}{*}{$\begin{array}{l}\text { Innovation talent culture } \\
\text { effect }\end{array}$} & \multirow{3}{*}{$\begin{array}{l}\text { innovation and expression of campus } \\
\text { students }\end{array}$} & the awards number of innovation competition \\
\hline & & the number of provincial honor or above \\
\hline & & the number of academic research achievements \\
\hline & \multirow{3}{*}{ achievements } & postgraduate qualifying \\
\hline & & employment rate \\
\hline & & the proportion of applicants and admissions in college entrance examination \\
\hline
\end{tabular}

III. MULTIPLE SYNTHETIC EVALUATION MODEL BASED ON AHP AND FUZZY MATHEMATICS

Multiple synthetic evaluation model based on AHP and fuzzy mathematics is a method which combined with AHP and fuzzy mathematics. It uses fuzzy membership theory to reasonably quantify qualitative index. So it solves the question of the combination with qualitative and quantitative evaluation. Currently, the method has been widely used in the evaluation of enterprise flexible index system, regional investment environment evaluation, job evaluation and so on.

The analysis process of multiple synthetic evaluation model based on AHP and fuzzy mathematics includes two parts. First step, establishing evaluation model with AHP and calculating the weight of each factor. Second step, evaluating with multiple synthetic evaluation method. Multiple synthetic evaluation model is based on AHP and supplement each other and jointly improves the scientific and reliability of the evaluation.

3.1 Determining the weighs of evaluation indicators in innovation talents training in universities by AHP

3.1.1 Determining the weigh of evaluation indicators by AHP 
a) Constructing judgment matrix

The element value of judgment matrix reflects people's understanding of the relative importance of various elements, such as strengths and weaknesses, preferences, strength and so on. It generally uses the ratio scale of 1-9 and reciprocal.
When the importance of each element can be obtained by the ratio with practical significance the corresponding element value of judgment matrix can obtain the ratio. The scale of Saaty is shown in Table 2.

TABLE 2. PROPORTION GRADE FOR RELATIVE SIGNIFICANCE

\begin{tabular}{|c|l|l|}
\hline Grade & \multicolumn{1}{|c|}{ Definition } & \multicolumn{1}{|l|}{ Explanation } \\
\hline 1 & Equal importance & The contribution of two factors is equal \\
\hline 3 & A factor is more important slightly than another factor & Experience and judgment slightly are favor to a factor \\
\hline 5 & Afactor is more important significantly than another factor & Experience and judgment obviously are favor to a factor \\
\hline 7 & A factor is more important strongly than another factor & Experience and judgment strongly are favor to a factor \\
\hline 9 & A factor is more important extremely than another factor & Experience and judgment strongly are favor to a factor \\
\hline $2,4,6,8$ & median of above two adjacent judgments & Experience and judgment extremely are favor to a factor \\
\hline
\end{tabular}

b) Determining the level weight

First calculating the component $w_{i}$ of $w$ by square root.

$$
w_{i}=\left(\prod_{i=1}^{n} b_{i j}\right)^{1 / n}, i=1,2, \cdots, n
$$

Second we made normalization processing to $W=\left(w_{1}, w_{2}, \cdots, w_{n}\right)^{T}$ and obtained the ranking weight vector $W^{0}=\left(w_{1}^{0}, w_{2}^{0}, \cdots, w_{n}^{0}\right)^{T}$.

$$
w_{A}=\sum_{i=1}^{n} w_{i}, w_{i}^{0}=w_{i} / w_{A} .
$$

Third calculating maximum characteristic root $\lambda_{\max }$ of A.

$$
\lambda_{\max }=\sum_{i=1}^{n} \frac{\left(B W^{0}\right)_{i}}{n w_{i}^{0}}
$$

Then consistency check must be made. We usually used random coincidence coefficient $C R$ to teat. $C R=C I / R I$, in which $R I$ was mean random consistency index value of judgment matrix shown in Table 3.

TABLE 3. VALUES LIST RI

\begin{tabular}{rl|l|l|l|l|l|l|l|l|l|}
\hline Dimension & 1 & 2 & 3 & 4 & 5 & 6 & 7 & 8 & 9 & 10 \\
\hline$R I$ & $\mathrm{n}$ & 0 & 0.58 & 0.9 & 1.12 & 1.24 & 1.32 & 1.41 & 1.45 & 1.49 \\
\hline & $C I=\frac{\left(\lambda_{\max }-n\right)}{n-1}$
\end{tabular}

When $\lambda_{\max }=n, C R=0$. But under the normal circumstances $\lambda_{\max }>n$, so $C I>0$. Usually $C R<0.1$, we believe the judgment satisfied, so the weight of layer indicators could be determined.

c) Determining the combination weights

Combination weight is the weight value which indicated the relative importance of the low-level indicators to the highest level indicators. This process was carried out from the top to the low layer. If higher layer A contained $\mathrm{m}$ factors, namely, $A_{1}, A_{2}, \cdots, A_{m}$, and its value of combination weight was $a_{1}, a_{2}, \cdots, a_{m}$, then the next layer $\mathrm{B}$ contained $\mathrm{n}$ factors, namely, $B_{1}, B_{2}, \cdots, B_{n}$, to the factor $A_{j}$, the layer weigh of B was respectively $b_{1 j}, b_{2 j}, \cdots, b_{n j}$. At this point, the $i$ th component of combination weight vector $\mathrm{T}$ of $\mathrm{B}$ layer was

$$
t_{i}=\sum_{j=1}^{m} a_{j} b_{i j}
$$

If consistency index of some factors of B layer was $C I_{\mathrm{j}}$ relative to the layer weigh of $A_{j}$, corresponding mean random consistency index was $R I_{\mathrm{j}}$, so random coincidence coefficient $C R$ of comprehensive weight of $B$ layer was

$$
C R=\frac{\sum_{j=1}^{m} a_{j} C I_{j}}{\sum_{j=1}^{m} a_{j} R I_{j}} .
$$

Similarly, when $C R<0.1$, we believed that the judgment matrix of combination weight had satisfied consistency, or needed to readjust the value of judgment matrix element.

3.1.2. Determining the weigh of evaluation indicators in innovation talents training in universities

According to expert advice we constructed judgment matrix for three indicators of rule layer, basis support strength, basis support contribution, innovation talent culture effect, as shown in Table 4.

TABLE 4. JUDGMENT MATRIX OF RELATIVE IMPORTANCE IN RULE LAYER INDICATORS

\begin{tabular}{|l|l|l|l|}
\hline$A$ & $A_{1}$ & $A_{2}$ & $A_{3}$ \\
\hline$A_{1}$ & 1 & $1 / 3$ & 2 \\
\hline$A_{2}$ & 3 & 1 & 4 \\
\hline$A_{3}$ & $1 / 2$ & $1 / 4$ & 1 \\
\hline
\end{tabular}

Then judgment matrix A was

$$
A=\left(\begin{array}{ccc}
1 & \frac{1}{3} & 2 \\
3 & 1 & 4 \\
\frac{1}{2} & \frac{1}{4} & 1
\end{array}\right) .
$$


We made normalization processing of column vectors and summed. We obtained matrix $(0.72,1.87,0.41)^{T}$.

Again we made normalization processing and obtained weight vector $W=(0.24,0.62,0.14)^{T}$.

$$
A W=\left(\begin{array}{ccc}
1 & \frac{1}{3} & 2 \\
3 & 1 & 4 \\
\frac{1}{2} & \frac{1}{4} & 1
\end{array}\right)(0.24,0.62,0.14)^{T}=(0.73,1.9,1.42)^{T}
$$

Calculating maximum characteristic root $\lambda_{\max }=3.03$, $C I=0.015$, so $C R=0.026<0.1$, which explained that judgment matrix had a certain degree of satisfaction and the weight value of three indicators of rule layer was more reliable, that is, weigh value was $W=(0.24,0.62,0.14)^{T}$, it passed the consistency test.

Similarly, we could get the judgment matrix of the target layer indicators and obtain the weight of every indicator in target layer and have passed the consistency test. Synthesizing calculation results, the weights of evaluation indicators in innovation talents training in universities were shown as Table 5.

TABLE 5. THE WEIGHTS OF EVALUATION INDICATORS IN INNOVATION TALENTS TRAINING IN UNIVERSITIES

\begin{tabular}{|c|c|c|c|}
\hline $\begin{array}{l}\text { The First Level } \\
\text { Indicators }\end{array}$ & Weigh & The Second Level indicators & Weigh \\
\hline \multirow{3}{*}{$\begin{array}{l}\text { basis support } \\
\text { strength }\end{array}$} & \multirow{3}{*}{0.24} & material basis & 0.32 \\
\hline & & subject foundation & 0.38 \\
\hline & & human resources & 0.30 \\
\hline \multirow{3}{*}{$\begin{array}{l}\text { basis support } \\
\text { contribution }\end{array}$} & \multirow{3}{*}{0.62} & material basis contribution & 0.25 \\
\hline & & teaching contribution & 0.48 \\
\hline & & scientific research contribution & 0.27 \\
\hline \multirow{2}{*}{$\begin{array}{l}\text { innovation talent } \\
\text { culture effect }\end{array}$} & \multirow[t]{2}{*}{0.14} & $\begin{array}{l}\text { innovation and expression of } \\
\text { campus students }\end{array}$ & 0.52 \\
\hline & & achievements & 0.48 \\
\hline
\end{tabular}

3.2. Determining the membership degree of evaluation indicators

There must were three basic elements to construct the model of fuzzy evaluation, namely factor set $U=\left\{u_{1}, u_{2}, \cdots\right.$, $\left.u_{n}\right\}$, judgment set $V=\left\{v_{1}, v_{2}, \cdots, v_{\mathrm{n}}\right\}$ and single factor decision. The decision of single factor expressed as a fuzzy map.

$$
\begin{gathered}
F: U \rightarrow F(V), u_{i} \rightarrow f\left(u_{i}\right) \equiv\left(r_{i 1}, r_{i 2}, \cdots, r_{i n}\right) \in F(V) . \\
R=\left(\begin{array}{cccc}
r_{11} & r_{12} & \cdots & r_{1 m} \\
r_{21} & r_{22} & \cdots & r_{2 m} \\
\vdots & \vdots & \cdots & \vdots \\
r_{n 1} & r_{n 2} & \cdots & r_{n m}
\end{array}\right) \in \mu_{m \times n} .
\end{gathered}
$$

We could induce a fuzzy transformation from the fuzzy matrix $R$.

$$
\tilde{T}_{R}: F(U) \rightarrow F(V), \quad A \rightarrow T_{R}(\tilde{A})=A \bullet R .
$$

$(U, V, R)$ constituted a fuzzy evaluation model. If we input a weight distribution $W=\left(w_{1}, w_{2}, \cdots, \mathrm{w}_{\mathrm{n}}\right) \in F(U)$ the model would export a comprehensive decision $B=W \bullet R=\left(b_{1}, b_{2}, \cdots, b_{m}\right) \in F(V)$.

$$
\begin{gathered}
\left(b_{1}, b_{2}, \cdots, b_{m}\right)=\left(w_{1}, w_{2}, \cdots, w_{n}\right)\left(\begin{array}{cccc}
r_{11} & r_{12} & \cdots & r_{1 m} \\
r_{21} & r_{22} & \cdots & r_{2 m} \\
\vdots & \vdots & \cdots & \vdots \\
r_{n 1} & r_{n 2} & \cdots & r_{n m}
\end{array}\right), \\
b_{j}=\underset{j=1}{\vee}\left(w_{i} \wedge r_{i j}\right), j=1,2, \cdots, m .
\end{gathered}
$$

In the process of evaluation, firstly according to the assessment results of evaluation team members to the people who were evaluated we calculated the proportion of the reviews level in second indicators and obtained the fuzzy matrix $R_{\mathrm{i}}$ corresponding to the second indicators. The fuzzy synthetic evaluation of the second level was the comprehensive evaluation of all indicators in first level.

$$
R_{i}=\left(\begin{array}{cccc}
r_{i 11} & r_{i 12} & \cdots & r_{i 1 m} \\
r_{i 21} & r_{i 22} & \cdots & r_{i 2 m} \\
\vdots & \vdots & \cdots & \vdots \\
r_{i j 1} & r_{i j 2} & \cdots & r_{i j m}
\end{array}\right) .
$$

Because many factors were taken into account the case of the weight distribution we could obtain fuzzy evaluation matrix $E$ on indicators by multiplication rule of fuzzy evaluation.

$$
E=\left(B_{1}, B_{2}, \cdots, B_{n}\right)^{T},
$$

where

$$
\begin{aligned}
B_{i} & =W_{i} \bullet R_{i}=\left(w_{i 1}, w_{i 2}, \cdots, w_{i k}\right) \bullet\left(\begin{array}{cccc}
r_{i 11} & r_{i 12} & \cdots & r_{i 1 m} \\
r_{i 21} & r_{i 22} & \cdots & r_{i 2 m} \\
\vdots & \vdots & \cdots & \vdots \\
r_{i k 1} & r_{i k 2} & \cdots & r_{i k m}
\end{array}\right) \\
& =\left(b_{i 1}, b_{i 2}, \cdots, b_{i m}\right) .
\end{aligned}
$$

Finally, we could judge the evaluation results according to the maximum membership degree principle. At the same time, based on evaluation results we could find the weak links of innovation talents training in universities and improve them.

\section{CONCLUSIONS}

The method of fuzzy synthetic evaluation had a lot of the advantages which previous evaluation methods had not. It 
was a more scientific and more reasonable evaluation method. By the use of evaluation model introduced in the paper we could easily carry out comprehensive evaluation to innovation talents training in universities and make the evaluation more scientific, more systematic. So the model had great promotion value. By mathematics method it made the questions which were compared very difficultly become more clarity and easier to compare. Because the thinking of the model was clear and specific we could prepare a computer program to simplify the calculation and analysis process although the calculation process was a bit complex. The model could improve evaluation efficiency and increase the fairness and impartiality of evaluation process. So the model had broad application prospects.

\section{ACKNOWLEDGEMENTS}

The paper was supported by Research Project in Education and Teaching of Hebei Unversity of Science and Technology (No. 2012-YB19) and the Social Science Foundation Project of Hebei Province (No. HB12JY050).

\section{REFERENCES}

[1] Schoessler, etc, "The Performance Appraisal as a Developmental Tool”, Journal of Nurses in Staff Development - JNSD, Vol 24 No. 3, pp. 12-18, 2008.

[2] Huanliang Xiong, and Canghai Wu, "The Study on Evaluation Indicators System of Case Teaching Based on AHP", Science and Technology Innovation Herald, No. 31, pp. 233-234, 2008.

[3] Shujun Cui and Liqian Wang, "The Evaluation Mode of University Employment Competitiveness Based on AHP”, Management Observer, No. 10, pp. 123-124, 2009.

[4] Ka-Chi Lam, Mike Chun-Kit Lam, Dan Wang, "MBNQA-oriented self-assessment quality management system for contractors: fuzzy AHP approach”, Construction Management and Economics, vol. 26, No. 5, pp.447-461,2008.

[5] Saaty.A.L., "Extending the Measurement of Tangibles to Intangibles”, J. of Informations Technology and Decision Making, Vol. 8, No. 1, pp. 7-27, 2009.

[6] Saaty.A.L., "Making decisions in hierarchic and network systems”, J. of Applied Decision Sciences, Vol.1, No. 1, pp. 24-79, 2008.

[7] Wu Qingwen, "Analysis on the Index System of Talent Training Evaluation in Study Colleges”, Journal of Academic Exchange, No.4, pp.186-191,2009.

[8] Ronald H Heck, “Administrative Effectives in Higher Education: Improving Assessment Procedure”, Research in Higher Educmion, Vol. 41, No. 6, pp. 25-29,2008.

[9] Ping Li, and Fachao Li,"Knowledge model based on decision tree and its application", Journal of Hebei University of Science and Technology, Vol. 30, No. 2, pp. 87-91, 2009.

[10] Bogacz,R, "Optimal decision-making theories : linking ncurobiology with behaviour”, Trends in Cognitive Sciences, No. 11, pp. 118-125, 2010 